\title{
The Data-Acquisition System of the KOTO Experiment
}

\author{
Chieh Lin, ${ }^{a, *}$ Mircea Bogdan, ${ }^{b}$ Yee Bob Hsiung, ${ }^{a}$ Qisen Lin, ${ }^{b}$ Yuting Luo, ${ }^{b}$ \\ Joseph Redeker, ${ }^{b}$ Yu-Chen Tung, ${ }^{a}$ Tong Wu ${ }^{a}$ and Yau W. Wah ${ }^{b}$ \\ ${ }^{a}$ National Taiwan University, \\ No. 1, Section 4, Roosevelt Rd, Da'an District, Taipei City, 10617, Taiwan \\ ${ }^{b}$ The University of Chicago, \\ 5801 S Ellis Ave, Chicago, IL 60637, United States \\ E-mail: jay@hep1.phys.ntu.edu.tw
}

\begin{abstract}
We present the latest and the near-future design of the KOTO data-acquisition system. The KOTO experiment is searching for the rare kaon decay $K_{L}^{0} \rightarrow \pi^{0} v \bar{v}$, which is sensitive to New Physics beyond the Standard Model due to the small theoretical uncertainty. In order to efficiently collect the candidate events under the high-intensity $K_{L}^{0}$ beam, the two-level trigger system was hence developed. The pulses from nearly 4000 channels were continuously digitized and pipelined with the depth of $5.2 \mu$ s at customized flush analog-to-digital converters (FADC) for trigger-processing. The level-1 trigger required the total energy in the calorimeter and the absence of hits in the veto counters. The level-2 trigger required two electromagnetic showers (clusters) in the calorimeter. The system dead time was measured to be $0.16 \mu$ s and the live time ratio was $99 \%$. In the near future, the customized module with multiple optical connectors will be used to collect all data from the FADCs and perform the event-building. The complete events are sent to the PC farms via $10 \mathrm{Gbps}$ throughput for more sophisticated trigger decisions. This not only improves the data collection efficiency but broadens the coverage of physics topics for the KOTO experiment.
\end{abstract}

40th International Conference on High Energy physics - ICHEP2020

July 28 - August 6, 2020

Prague, Czech Republic (virtual meeting)

\footnotetext{
${ }^{*}$ Speaker
} 


\section{The KOTO Experiment}

The KOTO experiment [1] at Japan Proton Accelerator Research Complex (J-PARC) [2] is searching for the $K_{L}^{0} \rightarrow \pi^{0} v \bar{v}$ decay. This decay mode is highly-suppressed and precisely-predicted by the Standard Model (SM) [3], and thus it is sensitive to New Physics. The experimental principle is shown in Figure 1. A bunch of protons first collides with the gold target and the secondary particles are guided to the KOTO beamline at $16^{\circ}$. The continuous proton collisions last for 2 seconds with the repetition of 5.2 seconds (spill). The KOTO detector is $21 \mathrm{~m}$ away from the target for the suppression of the short-lived particles. Moreover, the beam is purified by the photon absorber and the sweeping magnet, and narrowed by two collimators.

The $K_{L}^{0} \rightarrow \pi^{0} v \bar{v}$ reconstruction requires two photons hitting the endcapped calorimeter, which contains an array of un-doped cesium iodide crystals. Because the electromagnetic shower spreads the energy across the crystals, a photon hit manifests in a cluster. After measuring the energies and positions of the two photon clusters, the $\pi^{0}$ vertex and momentum are obtained by assuming that $\pi^{0} \rightarrow 2 \gamma$ occurs at the center of the beam. Due to the missing transverse momentum $\left(P_{T}\right)$ brought by neutrinos, the large $P_{T}$ of $\pi^{0}$ is required. Moreover, because the main $K_{L}^{0}$ decays are accompanied by charged particles or more than two photons, their suppression is achieved by the detection in the veto system that encloses the decay volume.

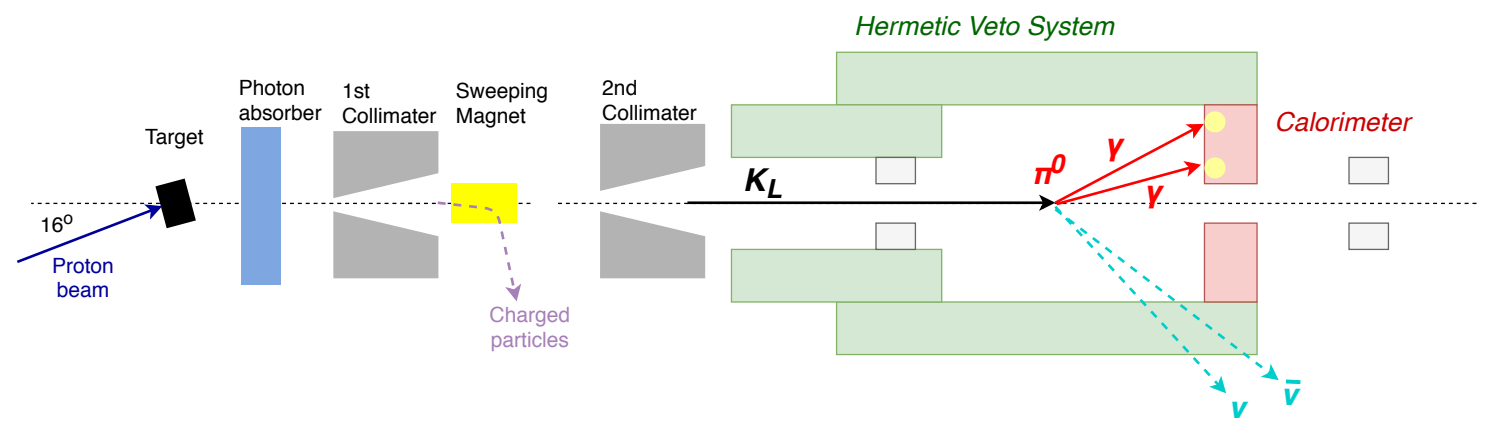

Figure 1: KOTO apparatus to search for the $K_{L}^{0} \rightarrow \pi^{0} v \bar{v}$ decay. The red (green) component shows the calorimeter (veto counters).

\section{Basic Concept of the KOTO Data-Acquisition System}

Based on the $K_{L}^{0} \rightarrow \pi^{0} v \bar{v}$ signature, two-level trigger system is introduced to elaborately collect the candidate events: level-1 trigger requires a finite energy deposit in the calorimeter (ET) and absence of hits in the veto counters (VETO), and level-2 trigger requires two photon clusters in the calorimeter. As shown in Figure 2, the pulses from all the readout components of the KOTO detector are continuously digitized by either $125-\mathrm{MHz}$ or $500-\mathrm{MHz}$ customized FADCs [4, 5]. The input data stream of the latest $5.2 \mu$ s is pipelined in the memory for processing a trigger decision. The features are first extracted from the pulses and sent to the core modules, which are responsible for trigger processing. After the calculation, a trigger is issued if an event fulfills all the conditions. Once a FADC receives the trigger, the pulses will be written out and eventually stored in the PC farms. 


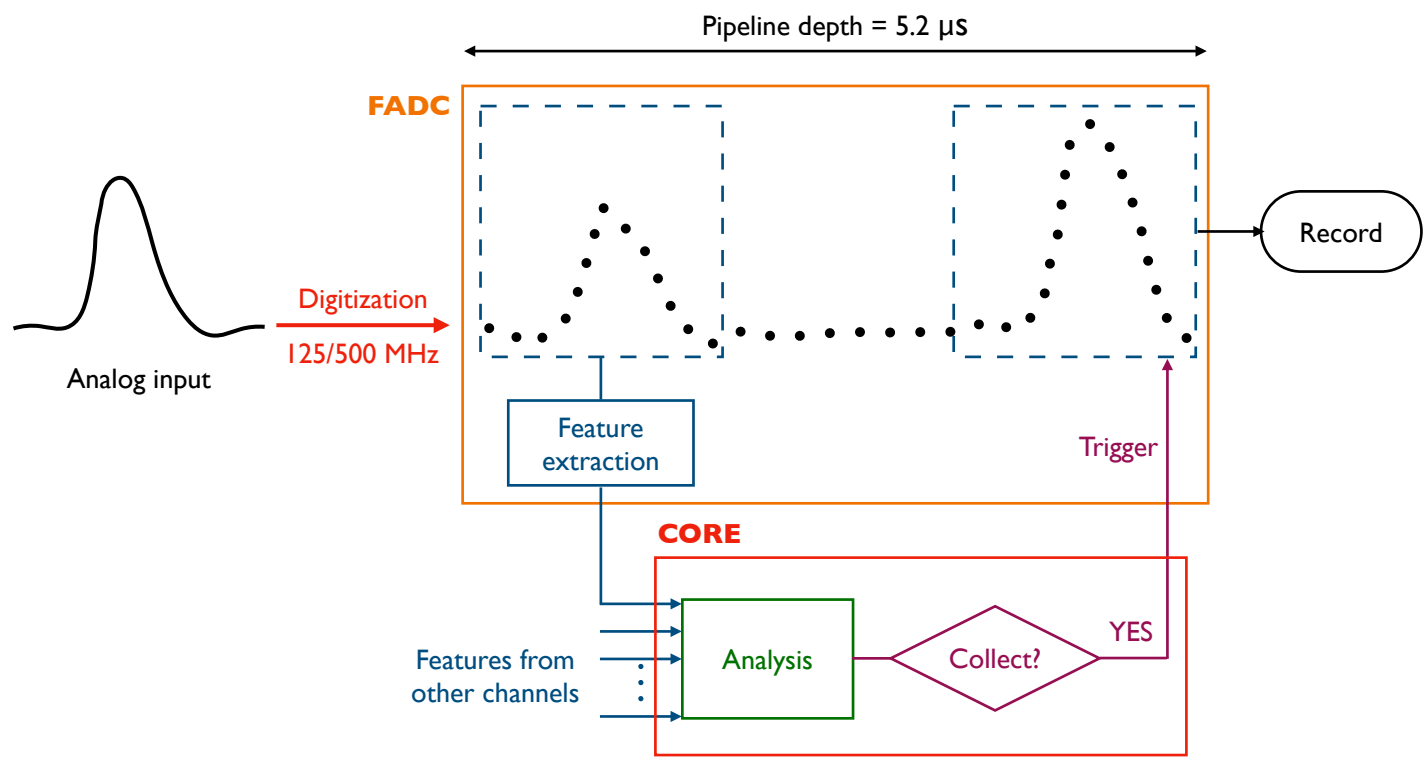

Figure 2: Trigger processing with the pipeline readout.

\section{Trigger Function}

The trigger calculation requires both energy and timing, which are the two fundamental quantities extracted from pulses. As shown in Figure 3, the baseline is first evaluated by finding the most-populated FADC count among thousands of samples. The pulse height is then evaluated by subtracting the baseline from the peak, which is by definition larger than the neighboring samples. A valid hit requires a peak inside the timing window. Because the energy is proportional to the pulse height, the energy of a hit can be calculated by a linear function, as shown in Figure 4.

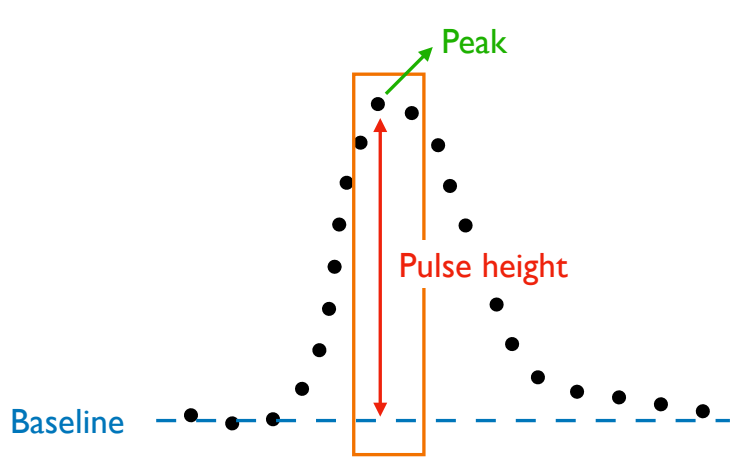

Peak timing window

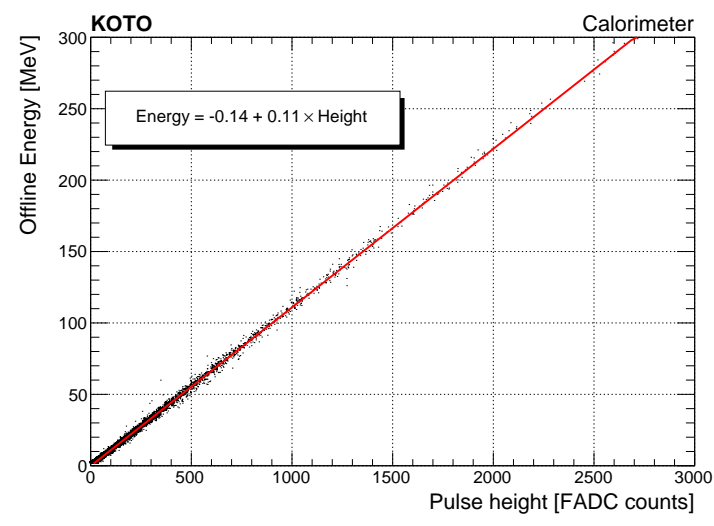

Figure 4: An example of a linear function fitted to a scattering plot of offline energy versus pulse height.

The level-1 trigger is evaluated every $8 \mathrm{~ns}$, governed by the sampling frequency. In the ET calculation, low-energy hits are skipped to inhibit the noise accumulation. The energy summation is 
realized by connecting several FADCs associated with the calorimeter in series, known as the daisy chain. The number carried over from the upstream board is added with its own result, then sent to the downstream board subsequently, until it reaches to the core module. In the VETO judgment, a veto decision is made when any hit exceeds the energy threshold. The hit multiplicity is also calculated by counting the number of hits in a subdetector.

The level-2 trigger is processed if an event satisfies the level-1 trigger condition. After the core module collects the hit map in the calorimeter, the cluster-finding is performed according to a high-speed algorithm counting the concavities and convexities of hit patterns [6]. The trigger is subsequently decided based on the resulting number of photon clusters.

The upgrade of the KOTO DAQ system was completed in 2018. Initially, the number of raw triggers, which were based on ET only, was $480 \mathrm{k} / \mathrm{spill}$. After the level-1 trigger selection, the rate was suppressed to $22 \mathrm{k} / \mathrm{spill}$. The level-2 trigger reduces the rate further to $13 \mathrm{k} / \mathrm{spill}$. The overall data loss due to a jam in trigger processing was estimated to be less than $1 \%$ in 2018 run, which was caused by the $0.16-\mu$ s dead time of the online cluster-finding.

\section{Performance}

Figure 5 shows the performance of the level-1 trigger. The data triggered only by ET was utilized to estimate the efficiency. For the ET efficiency plot, an exponential function was first fitted to the offline total energy distribution. The efficiency is then defined as the ratio of the data point to the fitted value. For the veto efficiency plot, the distribution of maximum energy among all channels in a veto counter was first obtained. Because the online veto decision was recorded in the data, the comparison to the distribution with the online veto implied the efficiency. Both ET and VETO efficiency plots were fitted with an error function. The offline energy threshold was $3 \sigma$ away from $\mu$, suggesting the loss was little.
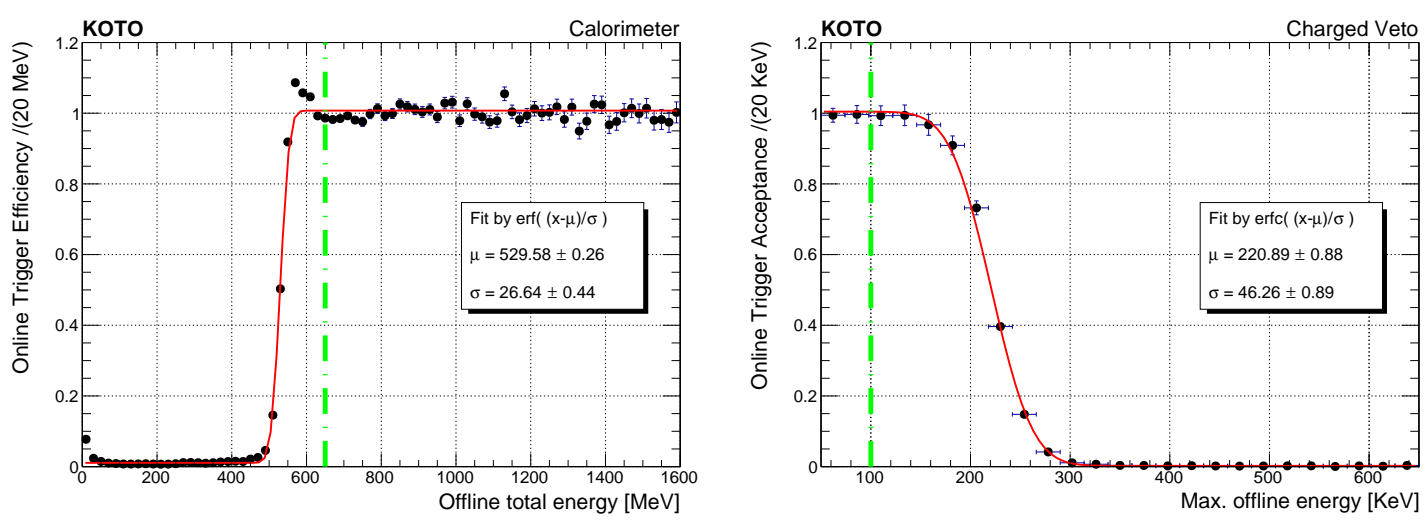

Figure 5: Efficiency curves of the total energy in the calorimeter (left) and the maximum energy among all channels in a veto counter (right). The red line shows the fitted error function and the green dashed line shows the offline energy threshold for $K_{L}^{0} \rightarrow \pi^{0} v \bar{v}$ analysis.

The performance of the level-2 trigger was also evaluated. By recording the online clustering result in the data, the level-2 trigger efficiency for the $K_{L}^{0} \rightarrow \pi^{0} \pi^{0}$ and the $K_{L}^{0} \rightarrow 2 \gamma$ decays were 
estimated. Meanwhile, the same algorithm and selection criteria were imposed on the GEANT4based Monte Carlo (MC) analysis with the pulse simulation. The comparable efficiencies were achieved, as summarized in Table 1 . The efficiency of the KOTO primary target $K_{L}^{0} \rightarrow \pi^{0} v \bar{v}$ decay was estimated to be $99.6 \%$, based on the Monte Carlo analysis.

\begin{tabular}{lll}
\hline Mode & $\epsilon_{\text {data }}[\%]$ & $\epsilon_{m c}[\%]$ \\
\hline$K_{L}^{0} \rightarrow \pi^{0} \pi^{0}$ & 96.8 & 96.7 \\
$K_{L}^{0} \rightarrow 2 \gamma$ & 99.6 & 99.2 \\
$K_{L}^{0} \rightarrow \pi^{0} v \bar{v}$ & - & 99.6 \\
\hline
\end{tabular}

Table 1: Level-2 trigger efficiencies of data $\left(\epsilon_{\text {data }}\right)$ and Monte Carlo simulation $\left(\epsilon_{m c}\right)$

\section{Future Plan}

After an accelerator upgrade in 2021, the beam intensity will be doubled. The level-3 trigger at the PC farms is hence proposed to additionally filter the candidate events, and might be practically managed to include other byproducts, such as the $K_{L}^{0} \rightarrow \pi^{0} e^{+} e^{-}$study. Because the level- 3 trigger algorithm typically requires a complete event for sophisticated analysis, the event-building is accomplished by the modules with multiple optical connectors [7] before sending data to PC farms, as shown in Figure 6. Those modules are arranged in a pyramid architecture. Sixteen FADC packages are first transmitted to a module at the speed of 2 Gbps and then assembled. There are eighteen such modules performing the local assembly in parallel, and the resulting packages are delivered to another module for the final assembly at the speed of 4 Gbps. A complete event is then constructed and sent to the PC farms via 10-Gbps throughput. By simulating the triggers with the real-beam structure at a doubled rate, the data loss due to a jam in bandwidth is estimated to be negligible.

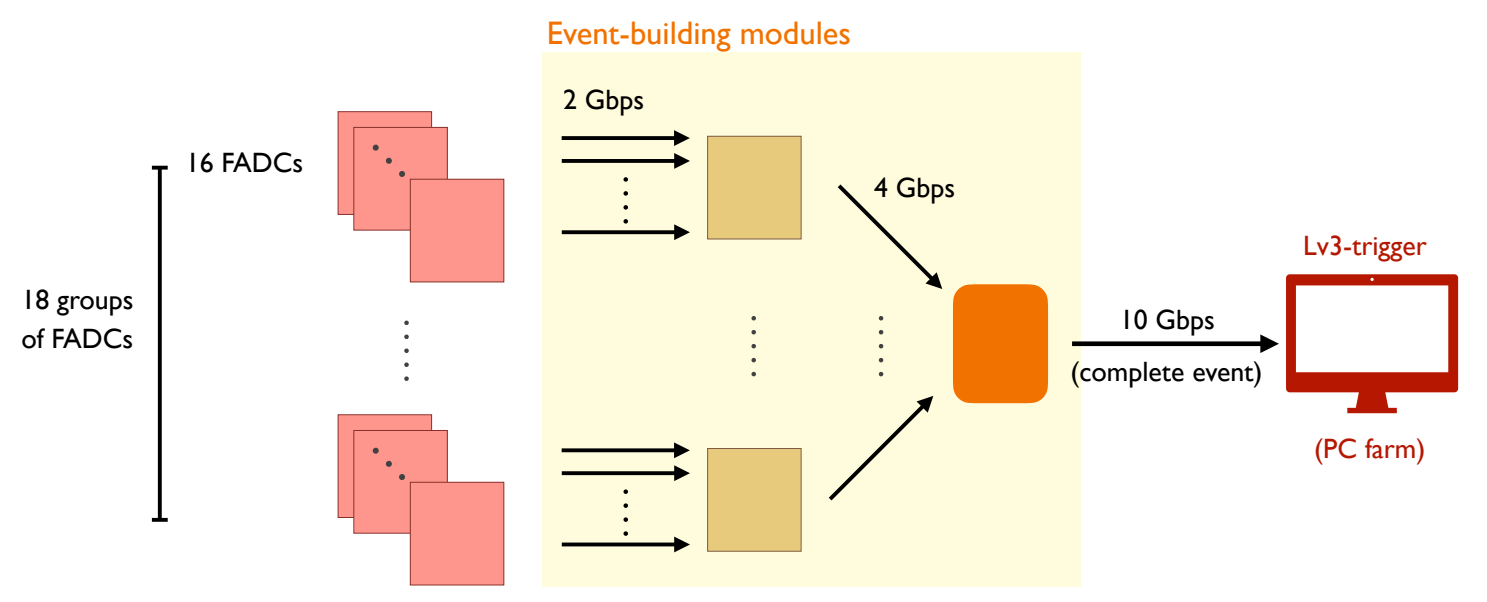

Figure 6: Architecture of the proposed level-3 trigger with the event-building modules beforehand. 


\section{Summary}

We upgraded the DAQ system for the KOTO experiment to collect the data efficiently under the high-intensity beam. The two-level trigger system with the pipeline readout at FADC enabled the explicit analysis of a candidate event. The trigger rate was suppressed from $480 \mathrm{k} / \mathrm{spill}$ to $13 \mathrm{k} / \mathrm{spill}$ in 2018 run, and both level-1 and level-2 triggers had the signal efficiency larger than 99\%. Also, the data loss due to the system dead time of $0.16 \mu$ s was less than $1 \%$.

We also considered an extension to DAQ system in the future. By introducing the eventbuilding modules and the level-3 trigger at the PC farms, the rate control can be enhanced with a negligible data loss under a doubled-intensity beam.

\section{References}

[1] T. Yamanaka and for the KOTO Collaboration, The J-PARC KOTO experiment, Progress of Theoretical and Experimental Physics 2012 (2012) .

[2] S. Nagamiya, Introduction to J-PARC, Progress of Theoretical and Experimental Physics $2012(2012)$.

[3] A.J. Buras, D. Buttazzo, J. Girrbach-Noe and R. Knegjens, $K^{+} \rightarrow \pi^{+} v \bar{v}$ and $K_{L}^{0} \rightarrow \pi^{0} v \bar{v}$ in the Standard Model: status and perspectives, Journal of High Energy Physics 2015 (2015) 33.

[4] M. Bogdan, J. Genat and Y. Wah, Custom DAQ Module for timing and energy measurements for J-Parc E14, 2009 16th IEEE-NPSS Real Time Conference (2009) 443.

[5] M. Bogdan, J. Genat and Y. Wah, Custom 12-Bit, 500MHZ ADC/Data Processing Module for the KOTO Experiment at J-Parc, 2010 17th IEEE-NPSS Real Time Conference (2010) 1.

[6] M.J. Haney, G.D. Gollin and T. Yamanaka, Cluster counting for E799/E832 at Fermilab, IEEE Conference on Nuclear Science Symposium and Medical Imaging (1992) 338.

[7] M. Bogdan, C. Lin, Y. Luo, Y. Tung and Y. Wah, Optical fiber center module for the KOTO experiment, Nuclear Instruments and Methods in Physics Research Section A: Accelerators, Spectrometers, Detectors and Associated Equipment 936 (2019) 340 . 